\title{
A Survey on Low Back Pain Risk Factors in Steel Industry Workers in 2015
}

\author{
Ehsan Rafeemanesh ${ }^{1}$, Farzad Omidi Kashani ${ }^{2}$, Reza Parvaneh ${ }^{1}$, Fatemeh Ahmadi ${ }^{1}$ \\ ${ }^{1}$ Department of Occupational Medicine, Mashhad University of Medical Sciences, Mashhad, Iran \\ ${ }^{2}$ Department of Orthopedic Surgery, Mashhad University of Medical Sciences, Mashhad, Iran
}

Study Design: This was a cross-sectional study.

Purpose: The aim of this study was to determine the prevalence of low back pain (LBP) and its association with individual factors and current job status among steel industry workers in Mashhad, Iran.

Overview of Literature: Several studies have been conducted on LBP and its related risk factors, some of which emphasized occupational factors as the main etiology of LBP. Meanwhile, individual risk factors have been emphasized in other studies. Despite several published articles, there are still many unresolved, basic issues about developing LBP.

Methods: For this study, 358 male workers were selected by a random sampling method and divided into two groups: production workers ( $n=201$ ) and administrative personnel ( $n=157)$. Data were collected using modified Nordic questionnaire and physical examination. Statistical analysis was performed to identify the correlation between individual factors and current job status with LBP. Results: Despite the young age of participants and their short employment duration, the overall prevalence of LBP was high (32.4\%) in this industry. The prevalence of non-specific LBP in production workers and administrative personnel was $26.8 \%$ and $21.0 \%$, respectively. Disk herniation was observed in $10.4 \%$ of production workers and $6.3 \%$ of administrative personnel. Age, employment duration, body mass index and smoking status were similar in the two groups. There was no significant relationship between LBP and current job status; however, a significant relationship was found between prevalence of LBP with age, duration of employment, and leisure time physical activity $(p<0.05)$.

Conclusions: We have not found any relationship between LBP and current occupational status suggesting that the effects of general health-related factors such as weight, age, leisure time physical activity, and duration of employment are more important than occupational factors in developing LBP.

Keywords: Low back pain; Occupational; Risk factors

\section{Introduction}

Low back pain (LBP) is the most common cause of disability in patients younger than 45 years of age and the second-most common reason for visiting a physician. LBP is also one of the most common work-related musculosk- eletal disorders (MSDs), the third-most common reason for surgery, and the fourth-most common reason for hospitalization [1].

Most people will experience back pain at some point in their life but in some occupational sectors such as industrial workers, nurses, and drivers, it is more widespread

Received May 25, 2016; Revised Jul 3, 2016; Accepted Jul 9, 2016

Corresponding author: Fatemeh Ahmadi

Occupational Medicine Department, Faculty of Medicine, Pardis, Azadi Square, Mashhad, Postal code: 9177948564, Iran

Tel: +9851-38002176, Fax: +9851-38002173, E-mail: Ahmadif@mums.ac.ir 
than other groups [2].

In view of occupational health, LBP is among the most important reasons for absenteeism, impairment, disability, and workers' compensation. Worldwide, LBP results in decreased productivity and imposes enormous costs upon the patient, family, society, industry, and the government [3].

We should consider that LBP is not merely a disease, but also it is a sign of several systemic, musculoskeletal, and neurological disorders [2].

Determining the main causes of LBP is often difficult, and in general there is little consensus on its etiology and even its definition [1].

LBP is a multifactorial disease and many risk factors may contribute to its development and process, including psychological, ergonomic, and physical factors [4]. Also, work environmental factors such as employment support, the number of employees, social support from colleagues, and overall job satisfaction are factors influencing LBP [2]. In spite of several articles published, there are still many unresolved, basic questions regarding the development of LBP and its prognosis.

Considering that MSDs are among the most common work-related issues in industry and lead to numerous problems in the work force, identifying casual factors may help prevent LBP development and complications. This study was done to assess the occupational and individual factors that influenced the development of LBP amongst steel industry workers in 2015.

\section{Materials and Methods}

This cross-sectional study was conducted in a steel industry in Mashhad, Iran. We selected 358 male personnel by random sampling method, and divided them into two groups: production workers (201) and administrative personnel (157). Production workers were mainly involved in lathing, welding, melting and assembling. This group was required to do heavy and very heavy physical activities, statically and dynamically for more than $70 \%$ of their working hours, including repetitive manual handling, prolonged standing, repeated bending from the waist, and repeatedly climbing stairs. A worker was considered to be exposed to prolonged standing if he spent more than $50 \%$ of the total working hours (more than 4 hours in our study) during a full work shift in a standing position. Heavy work refers to lifting up to a maximum of $45 \mathrm{~kg}$ with frequent lifting and/or carrying of objects weighting up to $23 \mathrm{~kg}$. Very heavy work refers to lifting more than $45 \mathrm{~kg}$ with frequent lifting and/or carrying of objects weighting $23 \mathrm{~kg}$ or more [5]. A job was considered to be repetitive when the duration of a task or group of tasks (a cycle) was less than 30 seconds. When cycles were longer than 30 seconds, the task was considered repetitive when the worker was performing the same motion for more than $50 \%$ of the cycle. Administrative staff mainly worked in administrative sections, and were sitting monotonously for at least $70 \%$ of their working time (at least 5.5 hours). The duration of each working shift in our study was 8 hours per working day.

The inclusion criteria were the employee's willingness to participate in the study along with at least one year of working experience at this plant. The exclusion criteria were a history of pre-employment LBP, traumatic spinal injury, a history of musculoskeletal disorders, and reluctance to participate in the study.

A modified Nordic questionnaire was used for collecting data in this study. The questionnaire consisted of three parts: (1) individual and demographic characteristics, including age, duration of employment, smoking and physical activity; (2) medical history; (3) history of LBP. The questionnaire was completed by a trained occupational health professional. In order to confirm a diagnosis in individuals reporting LBP, an occupational medicine specialist examined the patient.

LBP refers to "pain and discomfort localized below the costal margin and above the inferior gluteal folds, with or without leg pain" within the most recent 12 months. Patients who suffered from back pain were divided into two groups: (1) patients with non-specific low back pain (NSLBP), (2) patients with disk herniation. This classification was based on the patients' history, examination, MRI results (if applicable) and medical records.

Disk herniation was defined as a LBP causing leg pain, along with radiographic (MRI) evidence of disc herniation at the same side and level, leading to impingement, compression, or deviation of nerve tissue (as reported by a radiologist), or a patient with a valid medical certificate for disk herniation from a neurosurgeon or orthopedic surgeon. All steps of the study, including history taking, physical examination, and recording of the data were done by an occupational physician.

Non-specific LBP is defined as LBP that cannot be related to any specific disease such as fracture, infection, os- 
Table 1. Comparison of age, duration of employment and body mass index between two groups

\begin{tabular}{lccc} 
Variable & Administrative personnel & Production workers & $p$-value \\
Age $(\mathrm{yr})$ & $39.08 \pm 7.72$ & $38.76 \pm 5.87$ & 0.66 \\
Duration of employment $(\mathrm{yr})$ & $12.46 \pm 6.6$ & $13.49 \pm 4.68$ & 0.10 \\
\hline Body mass index $\left(\mathrm{kg} / \mathrm{m}^{2}\right)$ & $26.36 \pm 3.71$ & $26.35 \pm 3.32$ & 0.97 \\
\hline
\end{tabular}

Table 2. Comparison of non-occupational risk factors in patients with and without LBP

\begin{tabular}{|c|c|c|c|}
\hline Variable & Without LBP (n=242) & With LBP (n=116) & $p$-value \\
\hline Leisure time physical activity & & & $0.03^{*}$ \\
\hline No & $167(69)$ & $95(81.9)$ & \\
\hline Irregular & $53(21.9)$ & $16(13.8)$ & \\
\hline Regular & $22(9.1)$ & $5(4.3)$ & \\
\hline Overweight & $155(64.4)$ & $86(74.8)$ & $0.04^{*}$ \\
\hline Smoker & $18(7.4)$ & $8(6.9)$ & 0.85 \\
\hline
\end{tabular}

Values are presented as number (\%).

LBP, low back pain.

${ }^{*} p \leq 0.05$.

teoporosis, inflammatory or tumoral disease, or radicular syndromes.

Height was measured while the subject was standing against the wall with feet flat, his legs straight, arms at his sides, shoulders level, and looking straight ahead. Weight was measured without shoes and while wearing light clothes. Body mass index (BMI) was defined as the weight in kilograms divided by the square of the height in meters. In this study, we considered a BMI of more than 25 as overweight. Patients who smoked at least one cigarette per day were considered smokers and all others were considered non-smokers.

After collecting data, we entered the information gathered through observations, interviews, and clinical examinations into computers. Statistical analysis was done using SPSS ver. 11.5 statistical software. We used descriptive statistical methods, including central measures, measures of dispersion, and frequency distributions; and analytical statistical methods such as chi-squared test, $t$-test and logistic regression were used in data analysis as well. Data were considered statistically significance at $p<0.05$.

The protocol for this study was approved by the Ethics Committee of Mashhad University.

\section{Results}

Based on our analysis, the mean age of the subjects and

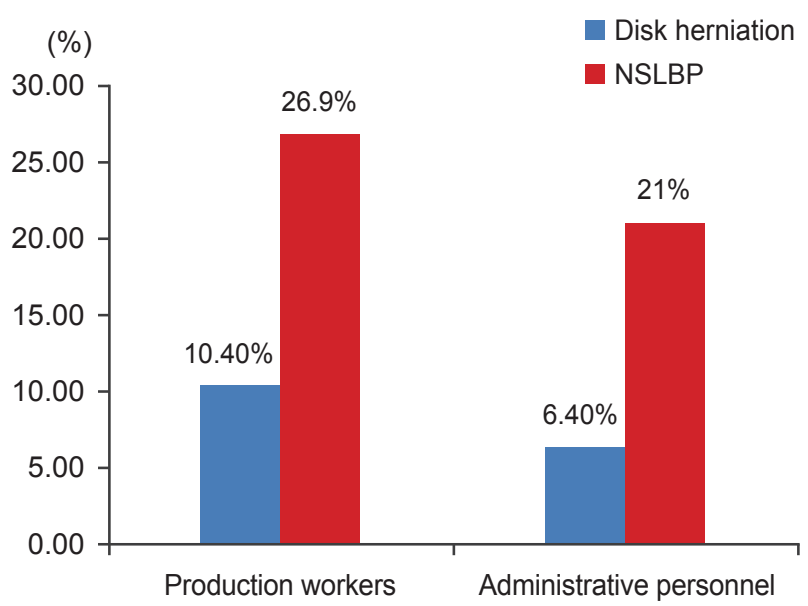

Fig. 1. Comparison of type of LBP between two groups. LBP, low back pain; NSLBP, non-specific low back pain.

the duration of employment was $38.9 \pm 6.73$ years and $13.04 \pm 5.70$ years, respectively.

As seen in Table 1, age, duration, and BMI were similar in production workers and administrative workers.

Two groups were not significantly different based on smoking status $(p=0.56)$. Fig. 1 shows the type of LBP in production workers and administrative workers. Based on our analysis, there was a significant relationship between LBP and age, BMI, duration of employment, and leisure time physical activity $(p \leq 0.05)$.

Table 2 shows the Comparison of non-occupational risk 
factors in patients with and without LBP.

To predict independent risk factors for LBP, the variables that showed a significant relationship with LBP (age, overweight, duration of employment and leisure time physical activity) were entered into the logistic regression model using the Backward method $\left(\mathrm{R}^{2}=0.04\right)$. Only the duration of employment was statistically significant and can be considered an independent risk factor $(p \leq 0.05)$. According to this analysis, each year of employment was associated with an $8 \%$ increase in the risk of LBP.

There was no relationship between LBP and known psychiatric disorders; 7.8\% (9) of workers with LBP and 8.7\% of workers without LBP had psychiatric disorders $(p=0.7)$.

\section{Discussion}

Despite the young age and short employment duration of this cohort, the 1-year prevalence of LBP was high in this industry (32.4\%). In a case-control study by Aghilinejad et al. [6] with 1,439 steel industry workers in Tehran, a high prevalence of musculoskeletal disorders was reported. Based on their study, the involved regions were most commonly back, knee and the neck. Also, they found a strong relationship of MSDs with BMI and the duration of employment.

In a case-control study conducted on 400 Indian steel workers in 2014, the overall prevalence of morbidities in the cohort was $60 \%$ and the highest morbidity was related to MSDs [7].

According to our findings, there is a considerable relationship between LBP and age. Similar results were obtained in previous studies $[4,8,9]$. The likely explanation for this association is that degenerative changes are hastened by the aging process [8]. Based on Sadeghian et al. [9], every 5-year increase in a worker's age correlates with a 1.46-fold increase in risk of LBP.

We showed that there is a negative relationship between LBP and leisure time physical activity. This was also observed in a cohort study by Mikkelsson et al. [10]. Physical activities can strengthen trunk muscles, and increase the body's endurance and movement abilities, resulting in better waist function $[10,11]$.

In our study, there was no relationship between LBP and smoking. This result is consistent with a study by Terzi and Altin [12]. However, in a meta-analysis by Shiri et al. [13], it was concluded that smoking cessation may reduce, but not completely eliminate, any excess risk; therefore they called smoking a "modest risk factor" for sciatica. However, there are other studies that reported smoking as an independent risk factor for LBP $[14,15]$.

According to the present study, there is a direct relationship between LBP and being overweight. Previous studies confirm the effects of this factor on intervertebral disc degeneration and the development of LBP $[16,17]$.

In addition, the results of our study showed that there is a significant relationship between LBP and the duration of employment. Similar results were obtained in previous studies $[2,4,8]$.

Based on our study, there is no association between the development of LBP and current job status. According to a systematic review by Kwon et al. [18], previous studies did not support the association between occupational physical activities and LBP. This finding may be due to poor quality scientific literature and the difficulty of determining the cause of LBP. In a meta-analysis by Taylor et al. [19], the incidence of LBP was similar in the community and in the occupational setting. Based on the results of this study, there are multiple diverse physical and psychosocial risk factors for first-time LBP. In another meta-analysis, there was a moderate association between mechanical exposures and LBP, although this association was complex [20].

Eight systematic reviews, using the Bradford-Hill causation criteria, concluded that it is unlikely that occupational sitting, standing, and walking, awkward postures, pushing or pulling, manual handling, lifting, bending and twisting, or carrying are independent risk factors for LBP [21-27].

However, Lis et al. [28] showed that occupational sitting for more than a half workday, in combination with awkward postures, increases the likelihood of LBP development.

Our study is a cross-sectional study; therefore, it is possible that any observed relationship between LBP and risk factors are not causal relationship.

Considering that age, BMI, duration of employment, and leisure time physical activity showed a significant association with LBP but none of them, except duration of employment, were independent risk factors for LBP, it appears that there may be other risk factors of LBP, such as psychosocial factors, that were not considered in this study. According to previous studies, psychosocial factors can influence LBP development and chronicity; however, their results are somewhat challenging [29,30]. Although 
our study didn't confirm a role of known psychiatric disorders in occupational LBP, patients were not specifically assessed according to psychosocial risk factors, which is a limitation of our study.

It seems clear that LBP is the result of the interaction of several occupational and non-occupational factors. In the future, longitudinal studies on relative importance of each individual risk factor and the effect of potential confounding variables are suggested to set a precedence for the prevention of LBP in the occupational setting.

\section{Conclusions}

We did not found any relationship between LBP and current occupational status, suggesting that the effects of general health-related factors such as weight, age, physical activity, and duration of employment are more important than occupational factors in the development of LBP. It is likely that lifestyle modification in combination with workforce education and ergonomic interventions will result in reducing the high rate of LBP and its related risk factors in this industry.

\section{Conflict of Interest}

No potential conflict of interest relevant to this article was reported.

\section{Acknowledgments}

This article is part of a research project entitled "A survey on low back pain risk factors in steel industry workers in 2015", enacted by Mashhad University of Medical Sciences in 2014 that was implemented with the support of Mashhad University of Medical Sciences.

\section{ORCID}

Fatemeh Ahmadi: orcid.org/0000-0001-5425-4557

\section{References}

1. Luke A, Ma CB. Back and lower extremity injuries. In: Ladou J, Harrison R, editors. Current occupational and environmental medicine. 5th ed. Philadelphia: McGraw-Hill Education; 2014. p.97-119.

2. Mohseni-Bandpei MA, Fakhri M, Ahmad-Shirvani
M, Bagheri-Nesami M, Khalilian AR. Risk factors for low back pain in nurses. J Mazand Univ Med Sci 2006;15:118-24.

3. Ahmadi H, Farshad A, Motamedzadeh M, Mahjob H. Epidemiology of low- back pain and its association with occupational and personal factors among employees of hamadan province industries. J Health 2014;5:59-66.

4. Habibi E, Pourabdian S, Atabaki AK, Hoseini M. Evaluation of Work-related Psychosocial and Ergonomics Factors in Relation to Low Back Discomfort in Emergency Unit Nurses. Int J Prev Med 2012;3: 564-8.

5. Palmer KT, Brown I. A general framework for assessing fitness for work. In: Palmer KT, Brown I, Hobson J, editors. Fitness for work. 5th ed. Oxford: Oxford University Press; 2013. p.1-20.

6. Aghilinejad M, Choobineh AR, Sadeghi Z, Nouri MK, Bahrami Ahmadi A. Prevalence of Musculoskeletal Disorders among Iranian Steel Workers. Iran Red Crescent Med J 2012;14:198-203.

7. Biswas M, Koparkar A, Joshi M, Hajare S, Kasturwar N. A study of morbidity pattern among iron and steel workers from an industry in central India. Indian J Occup Environ Med 2014;18:122-8.

8. Xu G, Pang D, Liu F, Pei D, Wang S, Li L. Prevalence of low back pain and associated occupational factors among Chinese coal miners. BMC Public Health 2012;12:149.

9. Sadeghian F, kalalian Moghaddam H, Javanmard M, Khosravi A, Adelnia S. An epidemiological survey of Low back pain and its relationship with occupational and personal factors among nursing personnel at hospitals of Shahrood Faculty of Medical Sciences. Iranian South Med J 2005;8:75-82.

10. Mikkelsson LO, Nupponen H, Kaprio J, Kautiainen H, Mikkelsson M, Kujala UM. Adolescent flexibility, endurance strength, and physical activity as predictors of adult tension neck, low back pain, and knee injury: a 25 year follow up study. Br J Sports Med 2006;40:107-13.

11. Hurwitz EL, Morgenstern H, Chiao C. Effects of recreational physical activity and back exercises on low back pain and psychological distress: findings from the UCLA Low Back Pain Study. Am J Public Health 2005;95:1817-24.

12. Terzi R, Altin F. The prevalence of low back pain in 
hospital staff and its relationship with chronic fatigue syndrome and occupational factors. Agri 2015;27: 149-54.

13. Shiri R, Karppinen J, Leino-Arjas P, Solovieva S, Viikari-Juntura E. The association between smoking and low back pain: a meta-analysis. Am J Med 2010; 123:87.e7-35.

14. Mikkonen P, Leino-Arjas P, Remes J, Zitting P, Taimela S, Karppinen J. Is smoking a risk factor for low back pain in adolescents? A prospective cohort study. Spine (Phila Pa 1976) 2008;33:527-32.

15. Capkin E, Karkucak M, Cakirbay H, et al. The prevalence and risk factors of low back pain in the eastern Black Sea region of Turkey. J Back Musculoskelet Rehabil 2015;28:783-7.

16. Shiri R, Karppinen J, Leino-Arjas P, Solovieva S, Viikari-Juntura E. The association between obesity and low back pain: a meta-analysis. Am J Epidemiol 2010;171:135-54.

17. Heuch I, Hagen K, Heuch I, Nygaard O, Zwart JA. The impact of body mass index on the prevalence of low back pain: the HUNT study. Spine (Phila Pa 1976) 2010;35:764-8.

18. Kwon BK, Roffey DM, Bishop PB, Dagenais S, Wai EK. Systematic review: occupational physical activity and low back pain. Occup Med (Lond) 2011;61:5418 .

19. Taylor JB, Goode AP, George SZ, Cook CE. Incidence and risk factors for first-time incident low back pain: a systematic review and meta-analysis. Spine J 2014; 14:2299-319.

20. Griffith LE, Shannon HS, Wells RP, et al. Individual participant data meta-analysis of mechanical workplace risk factors and low back pain. Am J Public Health 2012;102:309-18.

21. Roffey DM, Wai EK, Bishop P, Kwon BK, Dagenais S. Causal assessment of occupational sitting and low back pain: results of a systematic review. Spine J 2010;10:252-61.

22. Roffey DM, Wai EK, Bishop P, Kwon BK, Dagenais S. Causal assessment of occupational standing or walking and low back pain: results of a systematic review. Spine J 2010;10:262-72.

23. Roffey DM, Wai EK, Bishop P, Kwon BK, Dagenais S. Causal assessment of workplace manual handling or assisting patients and low back pain: results of a systematic review. Spine J 2010;10:639-51.

24. Roffey DM, Wai EK, Bishop P, Kwon BK, Dagenais S. Causal assessment of occupational pushing or pulling and low back pain: results of a systematic review. Spine J 2010;10:544-53.

25. Wai EK, Roffey DM, Bishop P, Kwon BK, Dagenais S. Causal assessment of occupational bending or twisting and low back pain: results of a systematic review. Spine J 2010;10:76-88.

26. Wai EK, Roffey DM, Bishop P, Kwon BK, Dagenais S. Causal assessment of occupational lifting and low back pain: results of a systematic review. Spine J 2010; 10:554-66.

27. Wai EK, Roffey DM, Bishop P, Kwon BK, Dagenais S. Causal assessment of occupational carrying and low back pain: results of a systematic review. Spine J 2010;10:628-38.

28. Lis AM, Black KM, Korn H, Nordin M. Association between sitting and occupational LBP. Eur Spine J 2007;16:283-98.

29. Nicholas MK, Linton SJ, Watson PJ, Main CJ. Early identification and management of psychological risk factors ("yellow flags") in patients with low back pain: a reappraisal. Phys Ther 2011;91:737-53.

30. Blyth FM, Macfarlane GJ, Nicholas MK. The contribution of psychosocial factors to the development of chronic pain: the key to better outcomes for patients? Pain 2007;129:8-11. 